Research Article

\title{
Region-Growing Algorithm on CT Angiography Images for Detection of Gynecological Malignant Tumor
}

\author{
Yufang Wen (iD, Dongfang Su $(D)$, and Qing Lin $(\mathbb{D}$ \\ Department of Obstetrics and Gynecology, People's Hospital of Xinzhou District, Wuhan 430400, Hubei, China \\ Correspondence should be addressed to Dongfang Su; 1400540115@xs.hnit.edu.cn
}

Received 22 June 2021; Revised 5 August 2021; Accepted 13 August 2021; Published 23 September 2021

Academic Editor: Gustavo Ramirez

Copyright (C) 2021 Yufang Wen et al. This is an open access article distributed under the Creative Commons Attribution License, which permits unrestricted use, distribution, and reproduction in any medium, provided the original work is properly cited.

\begin{abstract}
This paper aimed to explore pelvic lymphadenectomy for gynecological malignant tumors guided by computed tomography angiography (CTA) images under region-growing algorithm (RGA). 100 cases of malignant tumor patients who received pelvic lymphadenectomy in hospital from January 2018 to January 2020 were analyzed. Patients were classified into control group (CTA image) and experimental group (RGA-based CTA image), each with 50 cases. The overall accuracy (OA) of the pelvic CT image segmentation parameters under RGA, the watershed segmentation algorithm (WA), and the swarm intelligence optimization algorithm (SIOA) was compared. Comparisons of segmentation parameters, denoising performance, and CT imaging of patients as well as diagnosis rate and total efficiency rate were carried out. The results showed that overall accuracy (OA) of RGA was considerably higher versus watershed segmentation algorithm (WA) and swarm intelligence optimization algorithm (SIOA). However, false positive rate (FPR) and false negative rate (FNR) of RGA were greatly lower than those of other algorithms. RGA greatly improved the accuracy of pelvic tumor detection. The peak signal-to-noise ratio (PSNR) of RGA was superior to that of WA and SIOA, but differences in edge preservation index (EPI) value were not significant. The diagnosis rate of the experimental group was $48 / 50$ (96\%), while the diagnosis rate by manual means was 38/50 (76\%). For the diagnosis rate and total efficiency, results of the experimental group were evidently higher in contrast to the control group $(P<0.05)$. In conclusion, under RGA, CTA image-guided pelvic lymphadenectomy had good segmentation accuracy and denoising performance, and it was superior in total efficiency and diagnosis rate, which was worthy of clinical promotion.
\end{abstract}

\section{Introduction}

Malignant pelvic tumors are mainly manifested as pelvic masses. Ovarian tumors are the most common ones. In addition to the history of pelvic masses, they are also accompanied by lower abdomen enlargement, and CA125 is notably higher. B-ultrasound showed solid or mixed mass. About $1 / 2$ cases have a history of dysmenorrhea, and onethird of cases have menstrual disorders and delayed menstruation. In a few cases, there are no obvious symptoms. For areas that suggest no echo, in most cases, part B is mixed echo, coating thickness is irregular, with double-sided, and carbohydrate antigen 125 (CA125) increases. It is complicated to conduct anatomy of the pelvis. Similar to that of the abdomen, the pelvis has two main compartments: the peritoneal cavity and the sub-peritoneal space, which are separated by the peritoneum, and each compartment is a continuous space $[1,2]$. The sub-peritoneal cavity contains the entire abdominal and pelvic organs (and the respective mesenteric organs), extraperitoneal space ligaments, blood vessels, nerves, and lymphatic vessels.

Pelvic clearance (PE) refers to the radical lump resection of all lesions involving the pelvic organs, followed by pelvic reconstruction to restore the vital bodily functions that have been abolished. PE is a palliative therapy used to relieve symptoms of advanced pelvic cancer that cannot be controlled by radiotherapy. Due to the development of stable reconstructive surgical techniques and the improvement of perioperative treatments, parenteral surgery has developed rapidly. From palliative care alone to potentially curable surgery, among carefully nursed patients, the 5-year survival rate is over $50 \%$ confined to the pelvic refractory tumor 
$[3,4]$. Gynecological cancer is indication for PE. PE is an important task for both patients and doctors. Although the death rate associated with surgery is now less than $5 \%$, the death rate from serious complications after surgery is still more than $50 \%$. Therefore, pre-operative evaluation is necessary. PET/CT plays central role in pre-operative evaluation. It can locate, analyze, and image tumor tissue and retrograde, but it is not accurate. Since PET/CT has insignificant clinical treatment effects, RGA was introduced. The basic idea of the RGA is to combine pixels with similar attributes. For each region, a seed point growth should be specified as the starting point. Then, the pixel field around the seed point should be combined with the seed point, and the similarity point should continue to grow outward until no pixel contains a condition. For the RGA, selection of seed points should be carried out first. In many cases, the selection of seed points is carried out by means of human interaction $[5,6]$. For example, the interior of CT image is found as the extraction point, and then the growth criteria are confirmed, including gray value and color of color image, which are all about the expression between pixels. Finally, conditions were determined to facilitate the cessation of growth $[7,8]$.

In this work, PE and approach for different types of pelvic reconstruction and clinical analysis of pelvic lymphadenectomy for gynecological malignancies under the guidance of CTA image based on RGA were emphasized. $\mathrm{PET} / \mathrm{CT}$ was also reviewed as a roadmap for pre-operative treatment. In addition, how FDG PET/CT helps to identify changes in the treatment of persistent or recurrent tumors after radiotherapy was described, as well as how to avoid potential treatment risks due to previous radiation exposure. Finally, the expected post-management of the pelvis and the common appearance of post-PE complications were reviewed.

\section{Materials and Methods}

2.1. Research Subjects. One hundred patients with malignant tumors received pelvic lymphatic surgery in hospital from January 2018 to January 2020 were analyzed retrospectively. The median age of the patients was 58 years (29-85 years), and the median body mass index was $23.8 \mathrm{~kg} / \mathrm{m}^{2}$ $\left(16.0-40.3 \mathrm{~kg} / \mathrm{m}^{2}\right)$. Patients with CT serial images of pelvic cavity were included in the study. The main scanning parameters were $0.6 \mathrm{~mm}$ slice thickness and $0.6 \mathrm{~mm}$ slice distance. More than 900 12-bit slice images with $512 \times 512$ resolution were collected from each patient. The main difference between the two is that angiography is dynamic. After the contrast agent is pushed in, there is dynamic visualization in the target artery area, so the entire dynamic process can be seen very clearly. It is very intuitive and very accurate. Although it also pushes the contrast agent in CT, it scans it out layer by layer, so what can be seen is the plane diameter change map, which is equivalent to recreating the image of the morphological map like a movie. Although it is clearer, the accuracy rate is lower than that of angiography. In addition, angiography can be viewed from various angles during the operation. For example, if there is a serious stenosis which cannot be seen clearly at this position, the angle can be changed. Multiple angles are often used to find the lesion easily. CTonly presents a fixed axial position of the coronal plane for inspection, and it has no such function. For example, for intracranial aneurysms, 360-degree CT can be done, which can clearly determine the adjacency, size, and location of the aneurysm, and it is very beneficial for the next step of surgical planning. CT sequence of human abdomen scan is shown in Figure 1.

2.2. CTA Image Based on Region-Growing Algorithm. $R$ represents the whole image region, and the segmentation divides the region $R$ into $N$ sub-regions $R 1, R 2, \ldots R n$, and the following conditions need to be met.

(a) $\mathrm{U}(\mathrm{Ri})=R$.

(b) $R i$ is a connected region, $i=1,2,3, \ldots, n$.

(c) $R \mathrm{i} \cap R j=$ empty set, and for any $i, j, i \neq j$.

(d) $P(R i)=$ true, $i=1,2, \ldots, n$.

(e) $R(P i \cup R j)=$ false, $i \neq j$.

Region growth is the process of aggregating pixels or sub-regions into larger regions regarding a pre-defined criterion, with sufficient assurance that the segmented regions meet (a)-(e) criteria.

The region-growing algorithm mainly uses the determination of the growth seed point, the conditions of the area growth, and the stopping conditions of the area growth.

The number of seed points is chosen one or more times according to specific problems and can be determined completely automatically or through human-computer interaction according to specific problems. Region growth is actually some similarity criteria defined regarding the continuity between gray levels of pixels, and the condition of stopping region growth defines the termination rule. When no pixels meet the conditions for joining a certain area, the growth of the area will stop. In the algorithm, a variable is defined, and the maximum pixel gray distance is Reg_maxdist. When the absolute value difference between average gray value of all pixels in the area to be divided and gray value of the pixel to be added is $\leq$ Reg_maxdist, pixel will be added to the area to be divided. Instead, regiongrowing algorithm stopped, as shown in Figure 2.

In region growth, it is imperative to know the number of the pixel to be analyzed (represented by the $x$ and $y$ coordinate values of the pixel), as well as gray value of the pixel.

Determination of the growth criterion: it is supposed that coordinate position of the initial seed point is $(x 0, y 0)$, the pixel value of the initial seed point is $f(x 0, y 0)$, and the pixel value of the eight neighborhoods near the seed point is as follows.

$$
f(x i, y i)=f(x 0+\Delta x, y 0+\Delta y),(\Delta x, \Delta y \in[-1,1]) .
$$

Then, the growth criterion is as follows: 


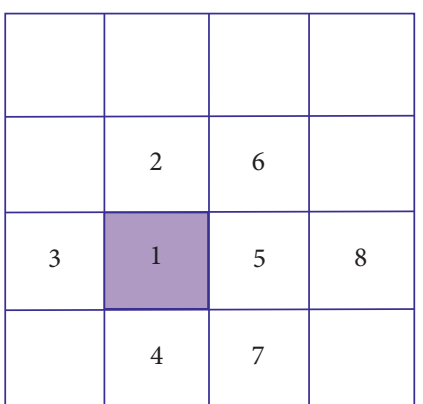

Dot label

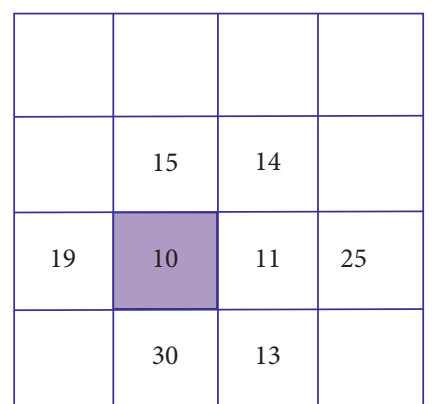

Gray value of the corresponding pixel

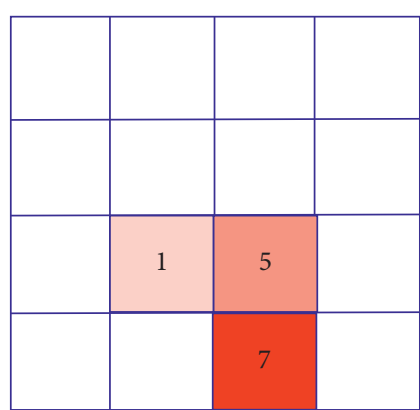

Direction of regional growth

FIgURE 1: Plain CT scan of human abdomen.

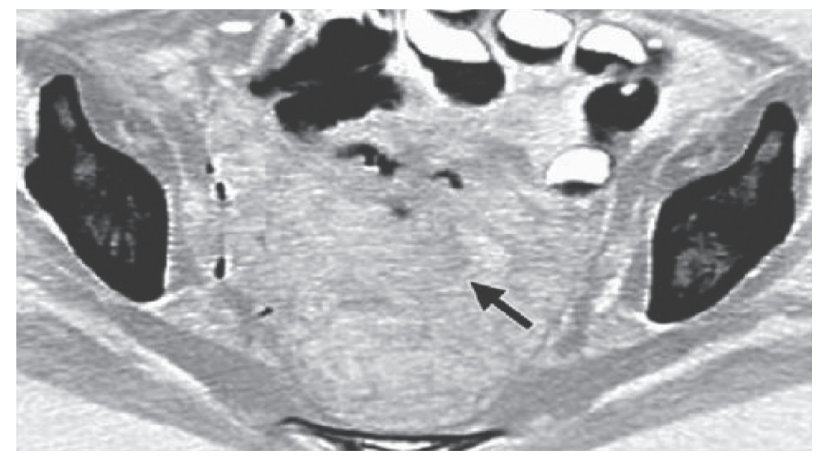

Figure 2: The direction of regional growth (from shallow to deep).

$$
f(x i, y i)= \begin{cases}0, & \{f(x i, y i) \notin \text { (lowthreshold, upthreshold) }\}, \\ 255, & \{f(x i, y i) \in \text { (lowthreshold, upthreshold) }\} .\end{cases}
$$

It can be seen from the above growth criterion that the selected range of the threshold in the region growth affects the segmentation result. This study was based on the CTA images. Due to the large difference in gray value of each region, CTA images combined with region growth can accurately segment the target region [5]. The details are shown in Figure 3.

2.3. Case Grouping. According to whether to perform RGA CTA imaging, all patients were classified as experimental group and control group equally. The specific patient information is shown in Table 1. The most common stage was cancer stage III (IIIC) according to International Federation of Gynecology and Obstetrics (FIGO) staging, and 50\% of patients were in IIIC stage. $20 \%$ of patients received any form of intestinal surgery. Splenectomy accounted for $3.5 \%$, and stoma accounted for $7.0 \%$. Average age was 58 years (29-85), and average BMI was $23.8 \mathrm{~kg} / \mathrm{m}^{2}$ (16.0-40.3).

In total, the median number of lymph nodes was 37 (5-76), 59 patients $(51.8 \%)$ had lymph node metastasis, and 5 lymph nodes (1-48) were positive. An average of 24.5 pelvic lymph nodes (2-52) were removed in 20 patients ( 2 of which were recommended by the American Cancer Society to remove only the para-aortic lymph nodes). Thirty-nine cases $(34.8 \%)$ were diagnosed with positive pelvic lymph nodes, including at least one lymph node (median: 3 cases, range: 1-26). 42 patients undergoing para-aortic lymphadenectomy were with 12 (1-48) lymph nodes. 51 patients (51\%) were with at least one positive para-aortic lymph node (median: 2 cases, range: $1-22$ ). Among the 65 patients with FIGO stage III tumors, 47 (47\%) patients had FIGO stage III tumors with positive lymph nodes, and 40 patients $(61.5 \%)$ had at least one positive para-aortic lymph node. Of the 14 patients with FIGO stage IV tumors, 12 (85.7\%) had active nodes, and 11 of them (11\%) had at least one metastasis to para-aortic nodes.

\subsection{Observation Indexes}

(I) Clinical symptoms: patient's signs of fever, diarrhea, vomiting, and coughing were observed and recorded.

(II) Imaging data: mainly CT images of the patient's chest were collected, and the region-growing algorithm was used to resegment the images to reacquire the characteristics of the patient's CT image.

(III) Treatment: all patients were treated with the same treatment plan for 2 weeks, to observe the recovery rate and total effective rate of patients.

2.5. Statistical Analysis. All data were statistically analyzed using SPSS22.0 software. Mean \pm standard deviation was how measurement data were expressed, tested by $t$-test. 

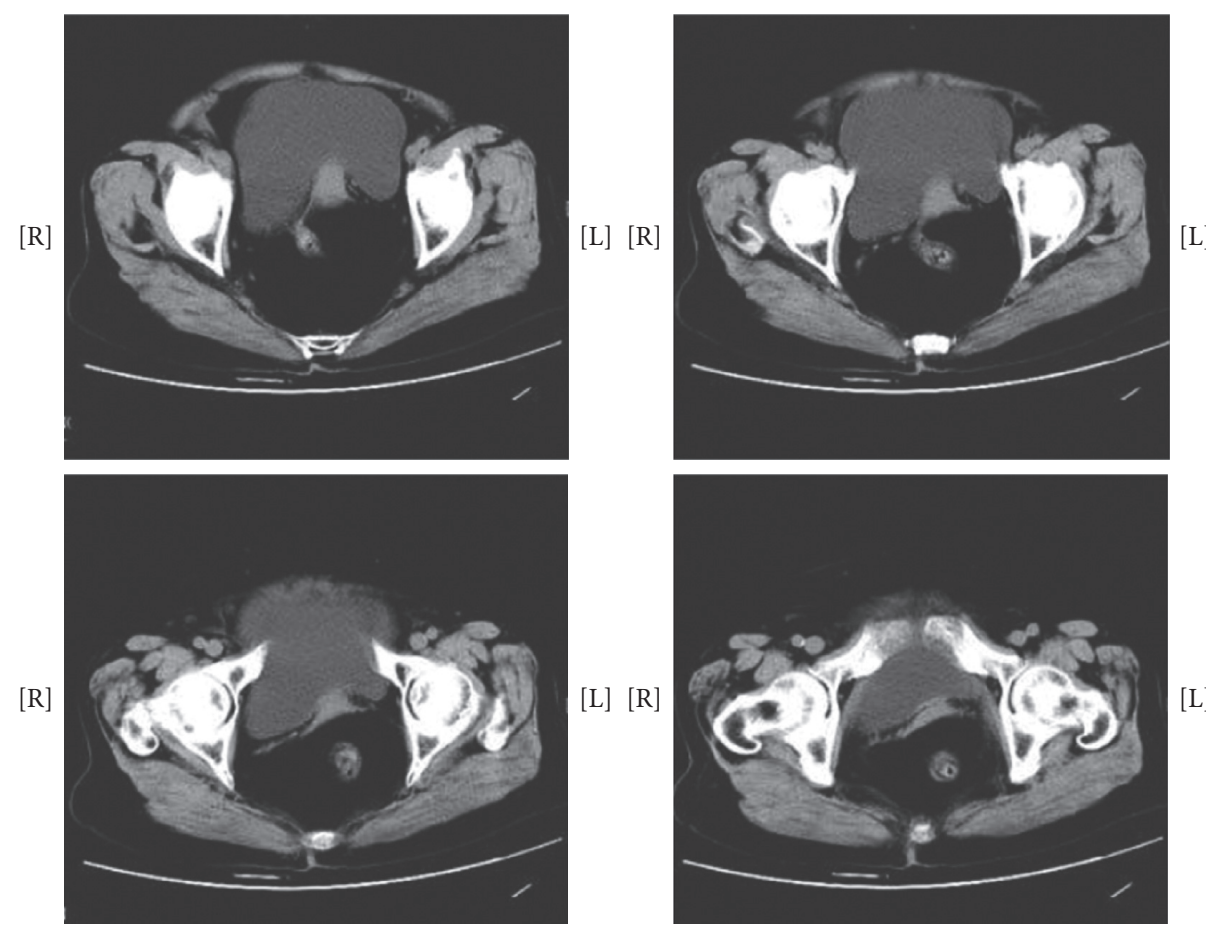

FIgURE 3: Unenhanced axial CT image.

TABLE 1: Information of patients.

\begin{tabular}{lc}
\hline Variable & \\
\hline Age (years) & $58 \pm 5$ \\
BMI $\left(\mathrm{kg} / \mathrm{m}^{2}\right)$ & $24.5 \pm 2.9$ \\
Staging laparoscopy & \\
$\quad$ No & $80(80 \%)$ \\
$\quad$ Yes & $20(20 \%)$ \\
Final tumor stage (FIGO) & \\
I & $6(6 \%)$ \\
II & $34(34 \%)$ \\
III & $30(30 \%)$ \\
IV & $30(30 \%)$ \\
ASA (American Society of Anesthesiologists) & \\
$\quad 1$ & $5(5 \%)$ \\
2 & $58(58 \%)$ \\
3 & $27(27 \%)$ \\
$\quad 4$ & $1(1 \%)$ \\
Unknown & $9(9 \%)$ \\
Histological grading & \\
G1 & $18(18 \%)$ \\
G2 & $19(19 \%)$ \\
G3 & $47(47 \%)$ \\
Unknown & $16(16 \%)$ \\
Histological type & \\
Serous & \\
Mucinous & \\
Endear cell & $45(45.0 \%)$ \\
Carcinosarcoma & $10(10 \%)$ \\
Other & $4(4 \%)$ \\
Ascites & $17(17 \%)$ \\
No & \\
Unknown & $42(42 \%)$ \\
& $48(48 \%)$ \\
\hline & $10(10 \%)$ \\
\hline
\end{tabular}

Percentage was how count data were expressed, tested by chi-square test. $P<0.05$ meant the difference was significant.

\section{Results}

3.1. Unenhanced Axial CT Image. The unenhanced axial CT image is shown in Figure 3.

3.2. Plain CT Scan of Human Abdomen. The plain CT scan of human abdomen is shown in Figure 1.

3.3. Comparison of Pelvic CT Image Segmentation Parameters with Different Algorithms. The results of pelvic CT image segmentation parameters of different algorithms are shown in Figures 4 and 5. The RGA had OA of 0.905, FPR of 0.0472 , and FNR of 0.0482 . Those of WA were $0.845,0.0865$, and 0.0845 , respectively. Those of SIOA were $0.827,0.1240$, and 0.0742 , respectively. It can be concluded that the OA of RGA was higher versus WA and SIOA, and both FPR and FNR were lower versus WA and SIOA. However, the differences of those values of different algorithms were not statistically significant.

3.4. Comparison of Pelvic CT Image Segmentation and Denoising Performance with Different Algorithms. The comparison of pelvic CT image segmentation and denoising performance of different algorithms is shown in Figure 6. PSNR of RGA was 27.826, and EPI of RGA was 0.925 . The PSNR of WA was 26.454, and EPI of WA was 0.968. The PSNR of SIOA was 26.871, and EPI of SIOA was 0.925 . The 


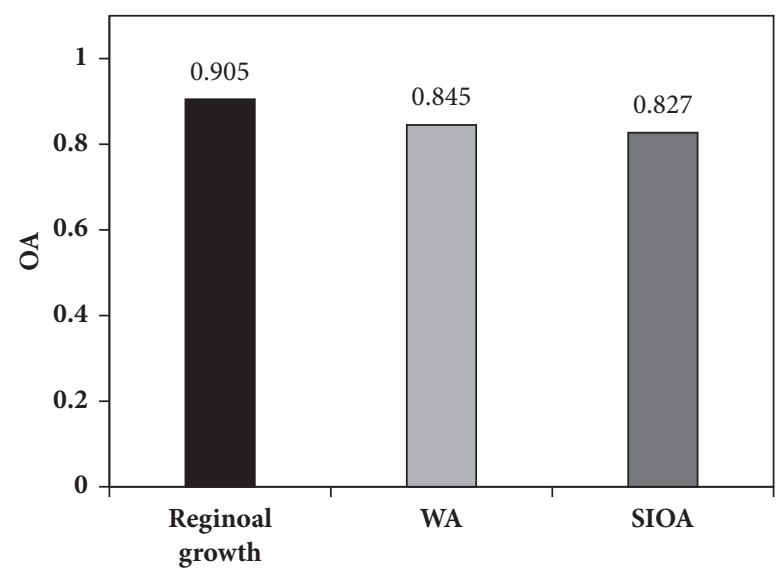

FIGURE 4: Comparison of OA values of different algorithms.

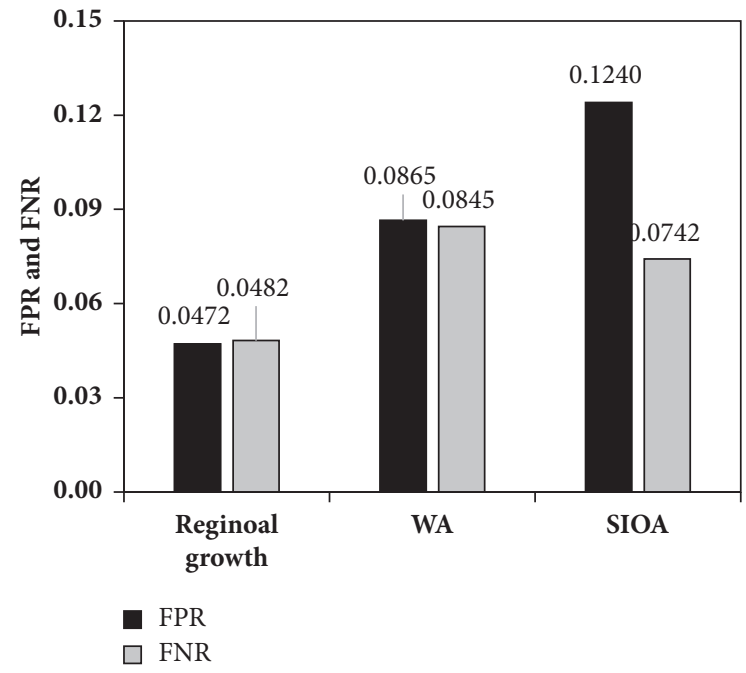

FIGURE 5: Comparison of FPR and FNR values of different algorithms.

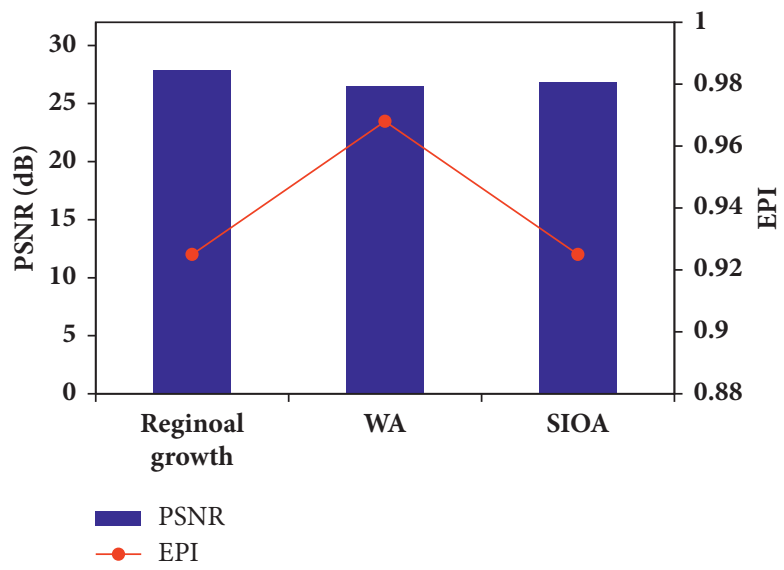

FIGURE 6: Comparison of denoising performance of different algorithms for pelvic CT image segmentation.

PSNR of RGA was higher than that of WA and SIOA. The EPI value of the WA was the highest, and those of RGA and SIOA were close to each other.
3.5. Analysis of CT Imaging Manifestations of Patients' Pelvic Cavity. The results of pelvic CT imaging of all patients were analyzed, and the number of main blood supply arteries, the 
TABLE 2: CT imaging findings of patients $(n=100)$.

\begin{tabular}{lc}
\hline Characteristics of feeding arteries (CT angiography) & Malignant \\
\hline$n$ & 100 \\
Number of main feeding artery branches & $10(10 \%)$ \\
1 & $35(35 \%)$ \\
2 & $20(20 \%)$ \\
3 & $35(35 \%)$ \\
4 & $68(68 \%)$ \\
Pattern associated with both route and newly formed vessels of the feeding artery & $32(32 \%)$ \\
$\quad$ Irregular & $49.45(11.5-70.00)$ \\
$\quad$ Regular & $45.25(7.80-62.30)$ \\
Distribution of newly formed blood vessels & $22(22 \%)$ \\
Tumor staining & $9(9 \%)$ \\
$\quad$ None & $18(18 \%)$ \\
Weakly positive & $51(51 \%)$ \\
Positive & \\
Strongly positive & \\
\end{tabular}

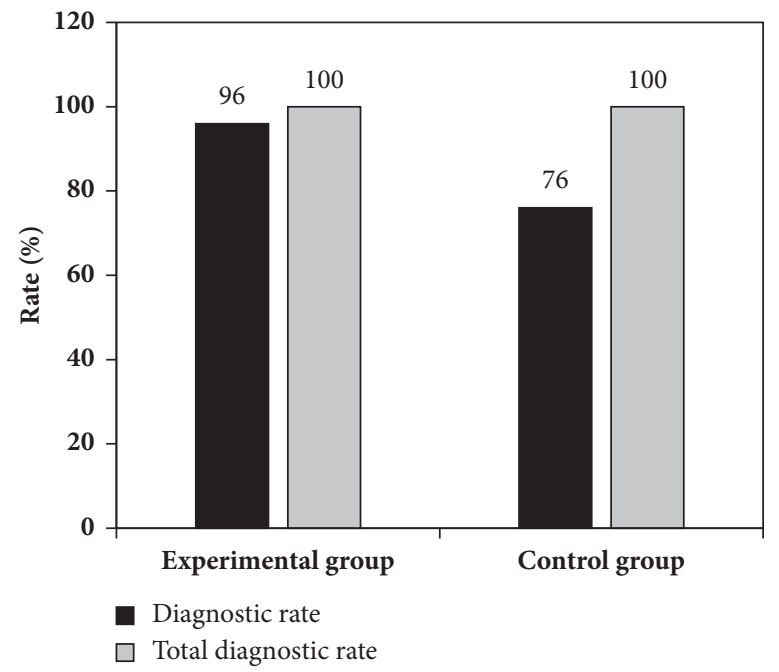

Figure 7: Comparison of diagnosis rate and total efficiency of patients between groups. ${ }^{*} P<0.05$ versus control group.

distribution of new blood vessels, tumor staining, and the blood supply arteries associated with routes and newly formed patterns are recorded in Table 2.

3.6. Comparison of Diagnosis Rate and Total Efficiency of Patients between Groups. The comparison results of the diagnosis rate and total effective rate of patients are shown in Figure 7. For the experimental group, CTA imaging based on the RGA was adopted, and for the control group, conventional CT imaging was adopted for manual diagnosis. As a result, the diagnosis rate in the experimental group was $48 / 50$ (96\%), while the manual diagnosis rate was $38 / 50(76 \%)$. The difference between the two groups was evident. The total effective rate of patients was $100 \%$.

\section{Discussion}

The CTA images based on the RGA can depict the outline of the tumor lymph node, which was supplemented by morphological post-processing modification, and satisfactory results were acquired. The results show that the CTA image under RGA had a high degree of specificity and a high positive predictive value, which was of great significance for the resection of pelvic tumors and lymph nodes.

In this study, CT pelvic images of different algorithms were compared, the OA value of the RGA was much higher than that of WA and SIOA, and FPR and FNP were far lower than those of the above two algorithms. It was proved that RGA greatly improved the accuracy of the pelvic tumor. Denoising performance of pelvic CT image segmentation was compared with different algorithms, the results still showed that the PSNR was superior to that of WA and SIOA, and the difference of EPI value was close, which was not evident [9-11]. In the comparative study of diagnostic rate and total efficiency, it was found that the results of CTA images based on RGA were much higher in the experimental group versus control group $(P<0.05)$.

In gynecological oncology, PE surgery is challenging, requiring extensive surgical personnel and fine operations. Factors that adversely affected the operation were the cause of recurrence of pelvic reconstruction, and impaired healing and immune response were caused by radiation therapy 
$[12,13]$. After surgery, the most common imaging complications are hematoma, abscess, lymphatic cyst, wound dehiscence, colorectal insufficiency, and urinary tract complications. Late post-operative complications include anastomotic stenosis, fistula, and tumor recurrence. Most of the recurrences of PE occur within 2 years, and most of them are pelvic masses or retroperitoneal masses or inguinal lymphadenopathy. In CT or MRI, new asymmetric soft tissue or enhancement should be considered with suspicion. Based on the RGA, CTA imaging can better detect recurrent diseases, which was superior to CT in the presentation of soft tissue. FDG PET/CT examination can also help diagnose the recurrence of the disease $[14,15]$. To distinguish recurrent tumors from post-operative tumor infection or inflammation, proper nursing should be performed. With limited treatment options and poor prognosis, surgical resection can still reduce the recurrence rate of the patient.

\section{Conclusion}

RGA had good accuracy and denoising performance in pelvic CTA image segmentation, which provided a positive effect for early diagnosis of gynecological malignant tumors and pelvic lymphatic treatment after resection. However, there are still certain limitations in this work. For example, the recovery effect presented by imaging was not analyzed in depth. The reason is that some patients have been discharged from the hospital, which makes it difficult to conduct followup review. Therefore, further research and analysis are needed.

\section{Data Availability}

The data used to support the findings of this study are available from the corresponding author upon request.

\section{Conflicts of Interest}

The authors declare that they have no conflicts of interest. dementia," Magnetic Resonance in Medicine, vol. 73, no. 1, pp. 102-116, 2015.

[6] B. I. Zeleniuk, L. V. Adamian, I. S. Obel'chak, and N. D. Khoroshun, "Role of multislice spiral computed tomographic angiography in the treatment of uterine myoma," Vestn Rentgenol Radiol, vol. 3, pp. 34-37, 2012.

[7] C. Meads, C. Davenport, and S. Małysiak, "Evaluating PET$\mathrm{CT}$ in the detection and management of recurrent cervical cancer: systematic reviews of diagnostic accuracy and subjective elicitation," BJOG, vol. 121, no. 4, pp. 398-407, 2014.

[8] Y. Jiang, M. Zhao, and C. Hu, "A parallel FP-growth algorithm on World Ocean Atlas data with multi-core CPU," The Journal of Supercomputing, vol. 75, pp. 732-745, 2019.

[9] H. Komatsu, M. Shimada, and D. Osaku, "Deep vein thrombosis and serum $\mathrm{D}$-dimer after pelvic lymphadenectomy in gynecological cancer," International Journal of Gynecological Cancer, vol. 30, no. 6, pp. 860-864, 2020.

[10] L. C. Wei, X. Li, and Y. Zhang, "Individualized pelvic lymphadenectomy should follow neoadjuvant concurrent chemoradiotherapy for locally advanced cervical cancer," Medicine (Baltimore), vol. 97, no. 14, Article ID e0331, 2018.

[11] P. E. Andersen, N. Lund, P. Justesen, T. Munk, B. Elle, and C. Floridon, "Uterine artery embolization of symptomatic uterine fibroida . Initial success and short-term results," Acta Radiologica, vol. 42, no. 2, pp. 234-238, 2001.

[12] E. M. Hecht, R. K. Do, S. K. Kang, G. L. Bennett, J. S. Babb, and T. W. Clark, "Diffusion-weighted imaging for prediction of volumetric response of leiomyomas following uterine artery embolization: a preliminary study," Journal of Magnetic Resonance Imaging, vol. 33, no. 3, pp. 641-646, 2011.

[13] Y. Tang, C. Chen, H. Duan, B. Ma, and P. Liu, "Low vascularity predicts favourable outcomes in leiomyoma patients treated with uterine artery embolization," European Radiology, vol. 26, no. 10, pp. 3571-3579, 2016.

[14] L. Ge, Q. Li, and J. Jiang, "Integration of nondegradable polystyrene and degradable gelatin in a core-sheath nanofibrous patch for pelvic reconstruction," International Journal of Nanomedicine, vol. 10, pp. 3193-3201, 2015.

[15] H. Liang, T. Ji, Y. Zhang, Y. Wang, and W. Guo, "Reconstruction with 3D-printed pelvic endoprostheses after resection of a pelvic tumour," Bone \& Joint Journal, vol. 99, no. 2, pp. 267-275, 2017.

\section{References}

[1] H. K. Pannu and M. Oliphant, "The subperitoneal space and peritoneal cavity: basic concepts," Abdominal Imaging, vol. 40, no. 7, pp. 2710-2722, 2015.

[2] A. Janvier, P. Rousset, J. Cazejust, O. Bouché, P. Soyer, and C. Hoeffel, "MR imaging of pelvic extraperitoneal masses: a diagnostic approach," Diagnostic and Interventional Imaging, vol. 97, no. 2, pp. 159-170, 2016.

[3] G. Baiocchi, G. C. Guimaraes, and R. A. R. Oliveira, "Prognostic factors in pelvic exenteration for gynecological malignancies," European Journal of Surgical Oncology, vol. 38, no. 10, pp. 948-954, 2012.

[4] E. J. Diver, J. A. R. Hain, and M. G. D. Carmen, "Total pelvic exenteration for gynecologic malignancies," International Journal of Surgical Oncology, vol. 2012, Article ID 693535, 9 pages, 2012.

[5] D. C. Alsop, J. A. Detre, and X. Golay, "Recommended implementation of arterial spin-labeled perfusion MRI for clinical applications: a consensus of the ISMRM perfusion study group and the European consortium for ASL in 